\title{
Imaginarios de naturaleza y el futuro de la biodiversidad
}

\author{
- Luis Germán Naranjo \\ WWF Colombia \\ Artículo de posesión para el ingreso como miembro correspondiente a la \\ Academia Colombiana de Ciencias Exactas, Físicas y Naturales el 20 de junio del 2019
}

\begin{abstract}
Resumen
La aceleración creciente de la crisis ambiental global demanda con urgencia respuestas contundentes de la comunidad de naciones y de la sociedad. A pesar del incremento de la conciencia colectiva acerca del deterioro de la biodiversidad y de los numerosos instrumentos, políticas acuerdos y convenios internacionales existentes para enfrentar este problema, los indicadores del estado de los ecosistemas, especies, poblaciones y servicios ecosistémicos continúan su declive incesante. Es necesario por lo tanto poner en marcha acciones distintas a las que hasta ahora han producido las numerosas iniciativas emprendidas durante los últimos cincuenta años, orientadas a la construcción de un poderoso imaginario social de naturaleza encarnado en todos los niveles de la sociedad.
\end{abstract}

Palabras clave: Biodiversidad; Imaginarios sociales; Ambientalismo; Conservación.

Nature imaginaries and the future of biodiversity

\begin{abstract}
The ongoing acceleration of the global environmental crisis calls for urgent and powerful responses both from the nations and the society. Despite the increasing consciousness about the deterioration of biodiversity, and the number of existing instruments, policies, agreements and international conventions to face this problem, indicators of the status of ecosystems, species and populations are in constant decline. It is thus necessary to initiate a new set of actions, different from those derived so far from the initiatives that have been launched over the last 50 years, aimed at the construction of a powerful social imaginary of Nature to be incarnated at all levels of society.
\end{abstract}

Key words: Biodiversity; Social imaginaries; Environmentalism; Conservation.

\section{Introducción}

A partir de la creación "oficial" del movimiento ambiental, con la celebración del primer día de la Tierra el 22 de abril de 1970, la sociedad global ha recibido advertencias cada vez más frecuentes y alarmantes sobre el deterioro de los ecosistemas, la disminución de poblaciones de vida silvestre, el número creciente de especies amenazadas de extinción y la pérdida progresiva de lo que hoy conocemos como servicios ecosistémicos. Pero hasta la publicación de los boletines de prensa que anunciaron el último informe del Panel Intergubernamental de Cambio Climático (Intergovernmental Panel on Climate Change, IPCC) a finales del 2018, los medios de comunicación masiva nunca habían divulgado la noticia de una fecha límite para una crisis que podría cambiar de forma irreversible las condiciones de vida en el planeta.

La información recopilada en dicho documento precisa que, a menos que la humanidad consiga disminuir en un $45 \%$ las emisiones de dióxido de carbono $\left(\mathrm{CO}_{2}\right)$ para el año 2030, será imposible cumplir con la meta de estabilizar el incremento de la temperatura global sin sobrepasar en más de $1,5^{\circ} \mathrm{C}$ el promedio de la era preindustrial (Intergovernmental Panel on Climate Change, 2018). Este límite se considera crítico, pues sobrepasarlo implicaría consecuencias tan devastadoras como la pérdida total de los arrecifes de coral, un incremento de $10 \mathrm{~cm}$ del nivel medio del mar, la alteración drástica en la distribución de cosechas tan importantes para la humanidad como el arroz, el maíz y el trigo y, desde luego, la modificación de la composición de biotas regionales como resultado de la desaparición de muchas especies y el desplazamiento geográfico de otras (Hoegh-Guldberg, et al., 2018).

\footnotetext{
Correspondencia:

Luis Germán Naranjo, lgnaranjo@wwf.org.co

Recibido: 8 de enero de 2019

Aceptado: 3 de mayo de 2019

Editor: Elizabeth Castañeda
} 
Por otra parte, este límite temporal permite abrigar la esperanza de que al fin se lograrán las respuestas contundentes que otros anuncios no han conseguido obtener del concierto de naciones, a pesar de su alarmante contenido. Por mencionar unos pocos casos sobresalientes, ni la evaluación de ecosistemas del milenio (Millennium Ecosystem Assessment Program, 2005), ni la determinación de la transgresión de los límites planetarios hecha por el Centro de Resiliencia de Estocolmo (Rockström, et al., 2009; Steffen, et al., 2015), ni el anuncio del inicio de la sexta extinción masiva de especies (Ceballos, et al., 2015), consiguieron poco más que una abundante cobertura noticiosa.

Si bien es cierto que, en gran medida, el cuerpo de información científica que documenta el cambio global ha sido responsable de los avances en materia de gestión ambiental a diferentes escalas, también lo es que la humanidad se encuentra lejos de asumir la responsabilidad que le corresponde en la protección y el manejo responsable del patrimonio natural. Como lo ha puesto de relieve la última versión del Informe Planeta Vivo (Grooten \& Almond, 2018), el continuo y marcado descenso de varios indicadores de la pérdida de la biodiversidad global con respecto a la línea de base de 1970, demuestra el alcance limitado de las acciones resultantes de los compromisos políticos de los países para frenarlo. Según lo señaló la Dra. Anne Larigauderie, Secretaria Ejecutiva de la Plataforma Intergubernamental Científico-normativa sobre Diversidad Biológica y Servicios de los Ecosistemas (Intergovernmental Science-Policy Platform on Biodiversity and Ecosystem Services, IPBES): “....el fracaso en asignar prioridad a políticas y acciones tendientes a detener y revertir la pérdida de diversidad biológica, y la continua degradación de las contribuciones de la naturaleza a las personas, pone en serio peligro las posibilidades de cualquiera de las regiones y de casi todos los países de lograr sus objetivos de desarrollo a nivel mundial" (IPBES, 2018).

Lo apremiante de estos nuevos llamados de alerta lleva a preguntarse cuáles son los impedimentos para lograr medidas mucho más efectivas de protección del patrimonio natural. Pues a menos que la humanidad logre eliminar dichas barreras, nos veremos abocados a la pérdida irreversible de las condiciones que hicieron posible su florecimiento.

\section{Hitos y limitaciones en la construcción del ambientalismo}

Desde la celebración del primer día de la tierra en 1970 hasta el final del siglo XX, la conciencia pública sobre los problemas ambientales creció de manera exponencial. Esto se tradujo en el desarrollo de una amplia y compleja legislación sobre el tema en la inmensa mayoría de los países, en el establecimiento de derechos y obligaciones ciudadanas, en la definición de funciones del Estado y de los organismos públicos responsables en materia ambiental, en la formulación y aplicación de políticas ambientales de distintas escalas y en la defensa del derecho ciudadano a un ambiente sano (Rodríguez-Becerra \& Espinoza, 2002). Este desarrollo es atribuible, en gran medida, a la serie de conferencias convocadas por la Organización de las Naciones Unidas (ONU) para abordar los problemas relacionados con el deterioro del patrimonio natural de la Tierra, en las cuales se empezó a construir paulatinamente la agenda ambiental global que desembocó en instrumentos tan importantes como las tres convenciones internacionales: Cambio climático, Diversidad biológica y Desertificación, formuladas en la cumbre de Río de Janeiro en 1992 (GuhlNannetti \& Leyva, 2015).

Sin embargo, una mirada retrospectiva a la relativamente breve historia del ambientalismo global también revela que, a pesar de los importantes logros conseguidos hasta ahora, las raíces de los problemas que se pretenden atacar siguen tan robustas y afianzadas como siempre. Por una parte, y como también lo señalan Guhl-Nannetti \& Leyva (2015), las convenciones originadas en la cumbre de Río han abordado los síntomas de los impactos antropogénicos sobre la naturaleza, pero no atacan sus causas. En consecuencia, los motores del cambio ambiental continúan operando y el deterioro de la biosfera se hace cada vez más profundo. De acuerdo con estimaciones recientes, menos de la tercera parte de las ecorregiones terrestres están relativamente libres de modificación antrópica, en tanto que la quinta parte se ve alterada extensivamente por la intervención del hombre y su fisonomía está dominada por asentamientos humanos, sistemas de producción agropecuaria, redes de infraestructura lineal y actividades industriales (Kennedy, et al., 2019).

La degradación y la pérdida de ecosistemas, sumadas a factores tales como la sobreexplotación, la expansión de especies invasoras y las alteraciones del clima a distintas escalas, continúan causando la disminución de un número cada vez mayor de poblaciones de vida silvestre. Entre 1970 y 2014 la abundancia promedio de 16.704 poblaciones de 4.005 especies de vertebrados declinó en un $60 \%$ (Grooten \& Almond, 2018). Esta reducción es un indicio del riesgo creciente de extinción de muchas especies: los valores actuales del Índice de la Lista Roja de especies de la Unión Internacional para la Conservación de la Naturaleza (UICN), correspondiente a los cinco grupos taxonómicos en los cuales se han evaluado todas las especies por lo menos dos veces (Aves, Mammalia, Amphibia, Anthozoa y Cycadaceae), van en disminución, lo cual refuerza la preocupación por la aceleración de las tasas de extinción de muchos tipos de organismos (Butchart, et al., 2018).

El desfase entre los instrumentos para abordar la problemática ambiental y los resultados conseguidos (Figura 1), también es evidente en otras escalas geográficas. A pesar de que la gesta ambientalista en América Latina siguió de cerca e incluso contribuyó a perfilar las tendencias globales en materia de conservación y uso sostenible de la biodiversidad (ver, por ejemplo, el papel visionario de la Convención del Hemisferio Occidental para la Protección de la Naturaleza 
y la Vida Silvestre, negociada bajo los auspicios de la Unión Panamericana en Rodríguez-Becerra \& Espinoza, 2002), su declinación en la región ha sido vertiginosa. La disminución media anual del índice planeta vivo para el Neotrópico entre 1970 y 2014 fue de 4,8 \% y, aunque su cálculo representa apenas las tendencias poblacionales de 1.040 poblaciones de 689 especies de vertebrados (Deinet, et al., 2018), una disminución total de $89 \%$ hace presumir que la situación de una de las biotas más ricas del planeta se encuentra en estado crítico.

Por último, el mismo desfase discutido para las escalas global y regional, se presenta a escala nacional $\mathrm{y}$ es particularmente notable en Colombia (Figura 2).
Además de haber contado con instrumentos legislativos y de política ambiental desde el inicio de la era republicana (Rodríguez-Becerra \& Espinoza, 2002), la nación ha sido una abanderada en materia de formulación de políticas ambientales, en paralelo con la evolución de distintos instrumentos en el plano internacional, como se ha puesto de relieve en el análisis histórico recogido en el Plan Nacional para la Gestión Integral de la Biodiversidad y los Servicios Ecosistémicos (Ministerio de Ambiente y Desarrollo Sostenible, 2012). Con la Constitución Política de 1991, el país elevó el manejo y la protección de los recursos naturales y el medio ambiente a la categoría de norma constitucional, es decir, se reconoció la obligación del Estado y

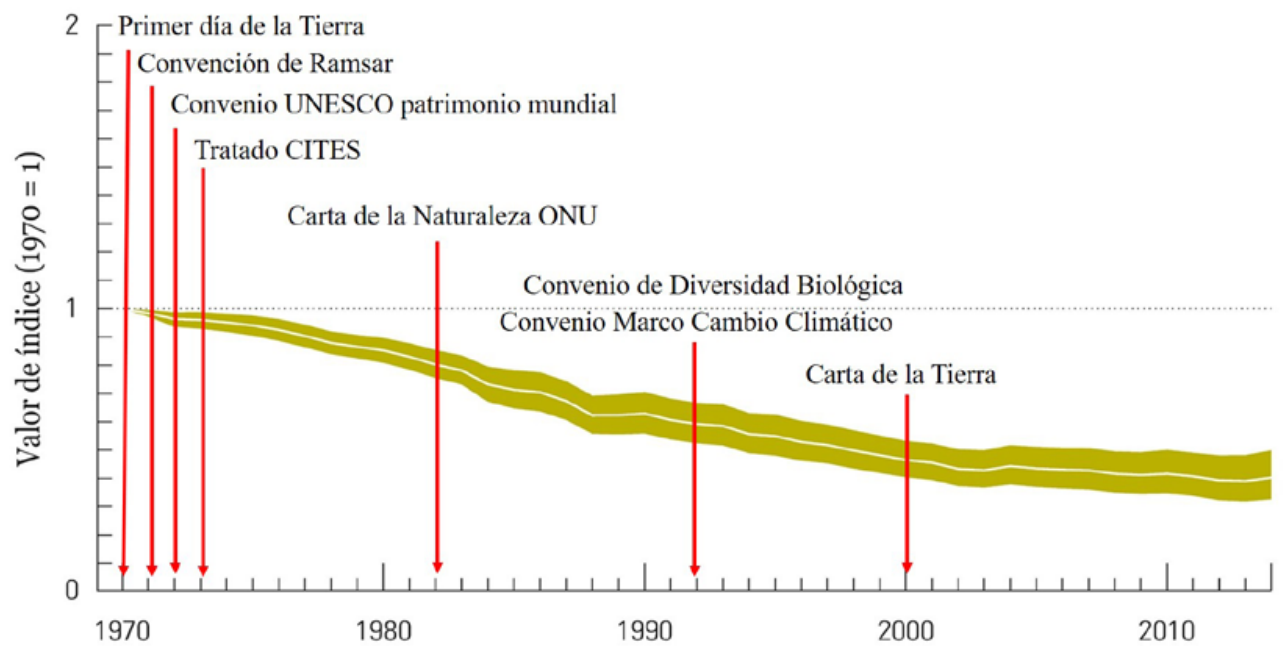

Figura 1. El desempeño limitado de la gestión mundial de la biodiversidad: algunos hitos históricos del ambientalismo global a partir de 1970 y el Índice Planeta Vivo. Fuente: elaboración propia a partir de la figura 20 en Deinet, et al. (2018).

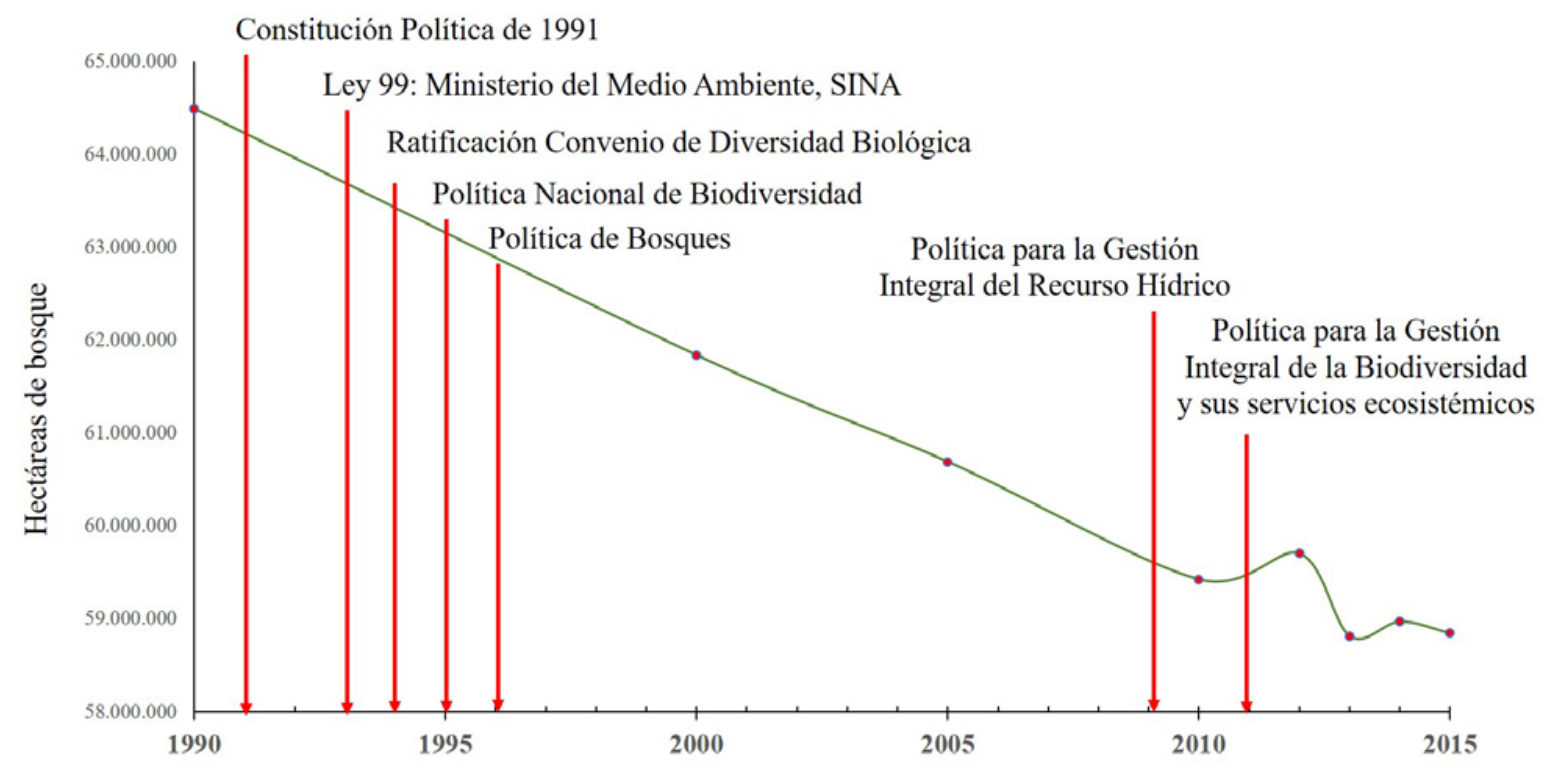

Figura 2. Pérdida de la cobertura forestal en Colombia desde 1990 y algunos desarrollos de la gestión ambiental en el país en el mismo período. Fuente: elaboración propia a partir de datos de IDEAM (2015). 
las personas de proteger las riquezas culturales y naturales nacionales, estableciendo el derecho de los colombianos a tener un ambiente sano y adoptando el desarrollo sostenible como modelo para orientar el crecimiento económico, el mejoramiento de la calidad de vida y el bienestar social de la nación. Desde entonces, es innegable que el estado colombiano ha hecho importantes esfuerzos para mejorar la gestión del patrimonio natural, entre los que se cuentan la creación y puesta en marcha de su sistema ambiental, la ratificación de numerosos acuerdos, convenios y convenciones internacionales para la conservación de la biodiversidad, el desarrollo de normas e instrumentos y el incremento significativo de la cobertura de sus áreas protegidas.

A pesar de estos avances, los indicadores recientes del estado de la biodiversidad colombiana son preocupantes. De los 323 ecosistemas continentales examinados en la actualización de la lista roja para el país, el $27 \%$ se encuentra en estado crítico, el $17 \%$ está amenazado y el $15 \%$ se considera vulnerable (Etter, et al., 2016). Según otros indicadores recogidos en el informe Colombia Viva (WWF-Colombia, 2017), la degradación y la pérdida de ecosistemas afecta por igual los ámbitos terrestre, marino y de agua dulce, lo cual se traduce en la pérdida del hábitat de muchas especies y, sumado a la sobreexplotación, desemboca en el incremento del número de especies amenazadas en aquellos grupos incluidos más de una vez en los libros rojos de la fauna y la flora colombianas: 54 en el nuevo libro rojo de los peces dulceacuícolas (Mojica, et al., 2012), 21 en el de reptiles (Morales-Betancourt, et al., 2015) y 27 en los de aves (Renjifo, et al., 2014, 2016). Hoy, el 2,22 \% de las especies presentes en el país se encuentra en una de las tres categorías de amenaza de la Unión Internacional para la Conservación de la Naturaleza (UICN).

\section{Visiones encontradas: ambientalismo y desarrollo}

Para entender el limitado alcance de los logros de la gestión ambiental a distintas escalas, es preciso examinar el contexto del desarrollo económico y social en el cual ha tenido lugar su evolución. En primer lugar, la explosión demográfica (de tres a seis millardos de personas en solo 50 años) y el rápido crecimiento económico (de 15 veces en ese mismo período) consecuencia del desarrollo técnico y científico posterior a la segunda guerra mundial, delimitaron el inicio de lo que hoy se conoce como la "gran aceleración del Antropoceno" (Steffen, et al., 2011a) y, al mismo tiempo, determinaron el advenimiento del movimiento ambientalista mundial. Por otra parte, los impresionantes logros de la revolución tecnológica en ese período (reducción de la mortalidad infantil, incremento en la productividad agropecuaria, mejoramiento de la asistencia médica, mayor afluencia económica, entre otros), contribuyeron a generar la noción del antagonismo entre el cuidado de la naturaleza y el desarrollo económico.

De alguna manera, esta aparente contradicción ha estado presente a lo largo de otros momentos históricos, pero desde el comienzo de la gran aceleración del Antropoceno ha sido más evidente y ha permeado las tres grandes etapas del ambientalismo moderno en la cultura occidental establecidas por Ramachandra Guha (Ulloa, 2002). En la primera de ellas, el manejo del patrimonio natural se centró en la preservación de espacios poco transformados y el desarrollo de estrategias para evitar la extinción de especies icónicas, y al estar disociado de los procesos de desarrollo económico, los sectores involucrados no consideraron las externalidades de los sistemas de producción. En este contexto, es obvio el conflicto entre la conservación y los sectores productivos cuyas actividades estaban basadas en la extracción de recursos, en la expansión de la agroindustria y en la construcción de grandes obras de infraestructura.

En la segunda etapa, marcada por el concepto de desarrollo sostenible producto del informe Brundtland (Comisión Mundial sobre el Medio Ambiente y el Desarrollo CMMAD, 1987), emergió la noción de que el bienestar de la sociedad dependía de los bienes y servicios que ofrece la naturaleza, aunque no con la fuerza suficiente como para que el sector productivo y el ambiental abandonaran sus respectivas orillas. Es cierto que en este período se establecieron los primeros controles y mecanismos de prevención de los efectos de la actividad antrópica en el ambiente, y que la sociedad civil incrementó su participación en los procesos de adopción de decisiones sobre el manejo del medio ambiente, pero la naturaleza siguió siendo vista, ante todo, como proveedora de recursos y materias primas.

Por último, la cooptación de la agenda ambiental por parte del modelo de desarrollo mercantilista global ha contribuido a hacer más profunda la brecha entre la conservación de la biodiversidad y el desarrollo económico, dado que el reduccionismo empresarial simplifica la visión integral y sistémica del ambiente en su afán por mejorar la competitividad y la productividad de los sectores productivos (Guhl-Nannetti \& Leyva, 2015).

A lo largo de las tres etapas prevaleció, entonces, una visión utilitaria de la naturaleza, herencia de la racionalidad occidental y manifiesta en una variedad de versiones según Rodríguez-Becerra \& Espinoza (2002): la minería de los recursos naturales renovables, el uso racional y la conservación de los recursos naturales, el conservacionismo, la gestión ambiental del Estado y el desarrollo sostenible. Todas ellas mantienen la división ontológica entre la naturaleza y la humanidad $\mathrm{y}$, al hacerlo, impiden la construcción de una relación entre sociedad y naturaleza en la que se entienda que el bienestar de los seres humanos depende del manejo respetuoso de los atributos y funciones de la biodiversidad.

No obstante, la evolución de los enfoques de conservación de la biodiversidad poco a poco ha dado cabida a enfoques holísticos que, de alguna manera, contribuyen a cerrar la brecha entre naturaleza y sociedad. Por una parte, la discusión acerca de los servicios ecosistémicos, intensificada a partir de la evaluación de ecosistemas del milenio (Reid, et al., 2005), llevó a considerar que ésta debía ir más allá de la utilidad y la racionalidad para trascender adecuadamente 
los valores socioculturales de los ecosistemas (Scholte, et al., 2015). Pero, además, el desarrollo del concepto de "panarquía", según el cual sistemas naturales, humanos y las combinaciones de ambos se entrelazan en ciclos adaptativos interminables de crecimiento, acumulación, reestructuración y renovación (Holling, 2001), ha conducido a entender los sistemas socioecológicos como unidades de análisis y manejo más aproximadas al estado actual de la biosfera.

\section{Lecturas de la naturaleza en el Antropoceno}

El reconocimiento del Antropoceno, la época geológica en la que las actividades de los seres humanos se han convertido en una fuerza geofísica, desató una oleada de propuestas para reorientar el rumbo de la sociedad global en su relación con el medio ambiente. Como lo plantearon Steffen, et al. (2011b), este reconocimiento es un recordatorio de que el Holoceno fue un período estable y propicio en el que las sociedades humanas, como las conocemos, se desarrollaron y florecieron, por lo que su disrupción actual demanda respuestas urgentes y contundentes: hemos ingresado a una nueva fase de la experiencia humana como habitantes de un mundo que será cada vez más diferente del que hoy conocemos.

El carácter global y la urgencia del cambio ambiental propio de esta época ha desembocado en la aceleración de la agenda internacional en torno a diferentes aspectos, como lo demostraron los acuerdos logrados en la cumbre de cambio climático de París en el 2015, aunque debe reconocerse que los avances en su gestión y refrendación han sido desiguales y no alcanzan la velocidad de respuesta requerida. Por otra parte, aunque la sociedad global está cada vez mejor informada acerca de esta problemática, el cuerpo de información que sustenta la búsqueda de soluciones mantiene la brecha entre la humanidad y el resto de la naturaleza, como veremos a continuación.

La enorme proporción de la superficie terrestre en donde la influencia humana sobre la fisonomía, la composición, la estructura $\mathrm{y}$, muy seguramente, la función de los ecosistemas es visible, según lo señalaran Vitousek, et al. (1997), llevó a algunos autores a considerar que la conservación, concebida como el mantenimiento de las condiciones "naturales" de un conjunto de ecosistemas, es una empresa poco menos que fútil, pues no es posible prometer el retorno de la Tierra a un estado prístino, propio de los paisajes previos al surgimiento de la humanidad (Kareiva, et al., 2007). Según estos mismos autores, una alternativa para frenar los impactos negativos de las acciones humanas sobre los ecosistemas debería basarse en el incremento de la domesticación de la naturaleza, lo cual podría, además, contribuir a incrementar el bienestar de la sociedad y fortalecer la economía global.

Este planteamiento condujo a una reacción airada por parte del "establecimiento" de la conservación (Soulé, 2013), basada en que aún había grandes segmentos del planeta mínimamente alterados y, por lo tanto, susceptibles de ser mantenidos como repositorios de la naturaleza que perdemos. Como epítome de este punto de vista, Wilson (2016) propuso la audaz idea de destinar la mitad de la superficie de la Tierra a la conservación estricta, como medida extrema para salvar la mayor cantidad posible de la biodiversidad restante. Pero, independientemente de los argumentos de uno y otro bando en esta controversia, resulta interesante observar que ambas orillas coinciden en apreciar el carácter ajeno de la humanidad con respecto a la naturaleza, pues mientras el primero la concibe de la manera utilitaria ya mencionada, el segundo la equipara a los remanentes de lo que fueran los paisajes y ecosistemas previos al desarrollo de las sociedades complejas.

Ante la encrucijada ambiental contemporánea y sus implicaciones para el bienestar humano, surge una tercera aproximación para enfrentarla a partir de lo que sus proponentes denominan los límites planetarios (Rockström, et al., 2009; Steffen, et al., 2015). Este marco conceptual define un espacio operativo seguro para la humanidad con base en la identificación de umbrales relacionados con procesos globales críticos, más allá de los cuales la humanidad no podría subsistir a la manera de las sociedades complejas existentes en la actualidad. A partir de tales umbrales se propone un enfoque de custodia planetaria basado en acciones simultáneas en múltiples escalas, la consideración de consecuencias ecológicas y socioeconómicas, los incentivos para el comportamiento de custodia, el fortalecimiento de las conexiones de la gente con los lugares que valora y el uso de transiciones demográficas como oportunidades de custodia (Chapin, et al., 2011).

A diferencia de las propuestas anteriores, la custodia planetaria plantea la idea de la dependencia de los seres humanos con respecto al resto de la naturaleza. Sin embargo, al centrar sus análisis en las condiciones que hacen posible la existencia humana como la conocemos, remite la gestión ambiental a un ámbito antropocentrista en consonancia con la visión de la naturaleza como espacio ajeno a lo que somos. Esta lectura, no obstante, es apenas una entre muchas que han circulado históricamente. En distintos momentos también se la ha vista como fenómeno colectivo del mundo, como esencia, cualidad o principio que informa acerca del funcionamiento del mundo, o como inspiración, guía y fuente de autoridad sobre los asuntos humanos (Coates, 1988). Con excepción de la última categoría, las demás consideran la naturaleza como un ente externo a la especie humana, y quizás por ello ha prevalecido la concepción de verla como el medio en el cual se inscribe su existencia. Entre las muchas limitaciones que entraña dicha lectura quizás la más importante es que la mayor parte de la humanidad, que debería estar involucrada en una decisión tan trascendental como la de escoger el futuro de su descendencia, queda excluida (Naranjo, 2016). En consecuencia, es importante desarrollar modelos que conduzcan a la construcción social de imaginarios de naturaleza para el Antropoceno. 


\section{Territorios, paisajes e imaginarios de naturaleza}

La idea de naturaleza que subyace las visiones utilitarias, así como gran parte de las propuestas y mecanismos desarrollados para enfrentar la crisis ambiental, son un legado de la modernidad que se sustenta en la aspiración a racionalizar la economía, así como en la eficacia y en lo instrumental y, en ese marco, el grado de desarrollo tecnológico se considera como medida del grado de autonomía de las sociedades humanas con respecto a las fuerzas de la naturaleza (Rivera, et al., 2006). Cuando esta se concibe principalmente como espacio físico para el usufructo, la optimización de su mantenimiento o la producción de determinado recurso, se dejan de lado o se ignoran imaginarios sociales en los que la realidad física y material del entorno tiene una poderosa carga simbólica.

Estas aproximaciones reduccionistas al medio ambiente son desafortunadas en la medida en que todo paisaje encarna la experiencia y las aspiraciones de sus habitantes y, por lo tanto, contiene núcleos de significado que expresan pensamientos, ideas y emociones. Un paisaje no solo nos presenta el mundo tal como es, sino que, de alguna manera, también es una construcción de ese mundo (Nogué, 2008) acorde con el sistema político en el que opera la cultura que lo construye, con sus patrones de uso de la tierra, sus preferencias estéticas y sus convenciones sociales (Nassauer, 1995).

El diálogo entre las sociedades y los paisajes hace posibles múltiples formas de entender la naturaleza y, en consecuencia, la coexistencia de dichas lecturas no debería verse como fuente de contradicciones y conflictos, sino como la oportunidad de construir nuevas relaciones con ella a partir de las dinámicas que tienen lugar entre las culturas territoriales y los paisajes donde se desenvuelven las sociedades. Después de todo, el sentido de lo real se apoya en un imaginario social constituido por imágenes de la realidad física y material del entorno en interacción con el sentido que se le da a dichas imágenes (Rivera, et al., 2006), $\mathrm{y}$ teniendo en cuenta que no solamente determina y crea una percepción de lo que es aceptado como real, sino que mantiene y cuestiona el orden social (Randazzo-Eisemann, 2012). En este sentido, sería posible replantear mediante la construcción de nuevos imaginarios de naturaleza, la visión utilitaria e instrumental responsable, en buena medida, del cambio global.

Por otra parte, dado que la comprensión del entorno es un fenómeno intersubjetivo en cuanto es compartido, experimentado e interpretado con otros y por otros, la construcción de nuevos imaginarios de naturaleza debe ser un ejercicio ampliamente participativo que propicie una nueva cultura del territorio, como lo ha propuesto ÁlvarezMunarriz (2011). Según este autor, tal proceso implica un modelo de interpretación del territorio que propende por la protección y gestión sostenible de los espacios que la sociedad considera valiosos a través de cambios en los estilos de vida y la implementación de acciones responsables e innovadoras.
La adopción de los imaginarios de naturaleza como condición de una nueva relación de la humanidad con el resto de los elementos que la componen es una propuesta tan ambiciosa como lo fueron en su momento el informe Brundtland (CMMAD, 1987) o la Carta de la Tierra (Earth Charter Initiative- ECI, 2000). Pero a diferencia de estos documentos, cuyos mensajes estaban dirigidos, ante todo, a los estados o a organizaciones de distinta índole, su concreción depende de una serie de condiciones cuyo cumplimiento trasciende la responsabilidad de dichos actores.

Por una parte, es imperativo lograr el reconocimiento general de que la humanidad no es un ente colectivo separado de la naturaleza. La admisión de que nuestra supervivencia y bienestar dependen de la funcionalidad de los ecosistemas y, por lo tanto, de las relaciones con otros seres vivientes, es un principio de la ética biocultural que transforma la pregunta filosófica central de cómo debemos vivir los seres humanos en la de cómo debemos cohabitar la Tierra (Rozzi, 2013).

En segundo lugar, es necesario replantear la valoración social de lo natural como aquello que no ha sido tocado por la acción humana (Nassauer, 1992). Cuando se equipara la noción de naturaleza con la idea de lo prístino, suele obtenerse una lectura equivocada de la integridad ecológica, pues es factible que un paisaje que parece natural no sea prístino, y que paisajes ecológicamente saludables no parezcan naturales, por lo cual la construcción colectiva de imaginarios de naturaleza debe considerar distintas formas de conocimiento, valores y prácticas ecológicas (Rozzi, 2013). Un diálogo intercultural en el que, además del conocimiento científico, intervengan otras lecturas de la naturaleza es fundamental en la interpretación de los atributos que un colectivo social considera esenciales y, por lo tanto, dignos de preservarse. Asimismo, contribuiría a armonizar las políticas y los instrumentos de gestión ambiental con las aspiraciones de quienes serán responsables de su ejecución (Rodríguez-Becerra \& Espinoza, 2002).

Un tercer requisito, relacionado con el anterior, es la comprensión del papel que juegan las formas de apropiación de la naturaleza en la formación y la consolidación de las identidades territoriales. Según Nogué (2008), la materialidad del paisaje está teñida de elementos intangibles que convierten cada lugar en algo único, lo cual conduce a la aparición de lo que conocemos como sentido de lugar. Los territorios adquieren una identidad singular ligada a la memoria colectiva, y ello constituye la base del apego local por una geografía, pues el sentido de lugar implica el aprecio local por las dimensiones estéticas, culturales y espirituales de los ecosistemas concebidas como servicios ecosistémicos valiosos (Chapin, et al., 2011). Desde este punto de vista, podría decirse que existen tantas naturalezas como identidades territoriales, lo que debería traducirse en propuestas de gestión medioambiental fundamentadas en la identificación de los elementos constitutivos de los sentidos de lugar. 


\section{Un momento para construir la naturaleza que queremos}

Teniendo en cuenta el carácter "multiescalar" de lo que sería un proceso de reformulación colectiva de imaginarios de naturaleza, conviene examinar su factibilidad en el contexto nacional colombiano. A lo largo de la historia del país, sus habitantes hemos entendido el territorio a partir de modelos simplificadores que no reconocen la complejidad y la vulnerabilidad de la base natural, y de ideas muchas veces lejanas, incluso opuestas a la complejidad de nuestro medio biofísico (Carrizosa, 2014). Aunque la revisión histórica de estas concepciones de la naturaleza en Colombia excede el alcance de este trabajo, el examen de algunas de ellas ofrece la posibilidad de determinar oportunidades y detectar obstáculos en la construcción de imaginarios que permitan mitigar y prevenir la erosión del patrimonio natural del país.

A partir de la bien conocida dicotomía en el modelo de apropiación del territorio iniciado con la conquista española, que privilegió la ocupación de las mal llamadas "tierras templadas" sobre la de los paisajes selváticos de las tierras bajas (Palacio, 2006), es posible reconocer algunos elementos que han determinado la configuración de las relaciones entre sociedad y naturaleza en Colombia. El primero de ellos consiste en la domesticación del paisaje como requisito para el desarrollo de la civilización. Esta noción, sin duda utilitaria, es responsable de la asimetría en la transformación de los paisajes colombianos, centrada en la planicie del Caribe y la región andina por sus condiciones climáticas más favorables a los españoles y la existencia de sistemas agrícolas y poblados indígenas en torno a los cuales establecer los primeros asentamientos coloniales (Carrizosa, 2014). La posterior ocupación de otros territorios, como los valles interandinos y sectores del piedemonte llanero, siguió este mismo modelo, buscando replicar sistemas de producción de ultramar en el proceso civilizatorio republicano.

La distinción entre la tierra domesticada y la naturaleza enemiga oculta formas complejas de apropiación social del paisaje que han persistido durante siglos y que responden a los factores ambientales determinantes propios de los espacios megadiversos de la selva lluviosa (Palacio, 2006), lo que favorece dos lecturas opuestas de dichos ambientes. La primera consiste en la visión de estos ecosistemas como fuentes de recursos en el marco de sucesivos modelos económicos extractivistas, como la explotación de la quina, el caucho, las pieles de animales silvestres, las maderas finas, los metales preciosos y los hidrocarburos. La segunda los contempla como espacios silvestres que albergan una rica biodiversidad, apreciada únicamente por los especialistas y por fuera de la cultura de masas (Andrade \& Castro, 2012).

La persistencia de estas lecturas es sin duda uno de los mayores obstáculos de la gestión ambiental en la Colombia contemporánea, pues la existencia de una periferia "natural", más allá del "centro" en el que se desenvuelve el país civilizado, es vista todavía como la oposición entre la anarquía y el caos frente al orden de la racionalidad (Serje, 2011). En el momento actual, cuando el país busca corregir el rumbo de su historia, estamos frente a una oportunidad única de romper este paradigma, para lo cual no solo es necesario promover un cambio cultural del significado de la biodiversidad en el territorio, como lo han señalado Sandino, et al. (2011), sino también emprender la construcción de un imaginario que permita una relación más armónica de la sociedad con el patrimonio natural de la nación.

Una tarea de estas dimensiones implica enfrentar una revisión crítica de los sistemas ambientales territoriales existentes (Carrizosa, 2014), cada uno de los cuales corresponde a la expresión paisajística de un determinado sentido de lugar, así como analizar las transiciones ecológicas de regiones reconocidas por su gran biodiversidad y su fragilidad ambiental, cuya verdadera dimensión solo podrá expresarse a mediano y largo plazo (Andrade, et al., 2018). Pero, además de estos insumos, que sin duda demandan el involucramiento de actores con conocimiento y experiencia analítica acordes con el tamaño del problema, el más importante requisito consiste en la popularización de ese conocimiento, de forma tal que promueva una participación social sin precedentes para convertir la conciencia de la responsabilidad individual frente al resto de la naturaleza en el más importante elemento de la tan anhelada y siempre elusiva "colombianidad".

\section{Conclusiones}

A pesar de las múltiples acciones que ha emprendido la humanidad desde los albores del ambientalismo para frenar la pérdida de la biodiversidad global, el deterioro del patrimonio natural es cada vez más acelerado, pues las convenciones y acuerdos internacionales relacionados con esta problemática apenas han abordado los síntomas del impacto antropogénico sobre la naturaleza, pero no sus causas. El desfase entre los instrumentos de política ambiental y los resultados conseguidos es evidente en distintas escalas geográficas.

El limitado alcance de la gestión ambiental es, en gran medida, una consecuencia del antagonismo cultural entre el cuidado de la naturaleza y el desarrollo económico. La visión utilitaria de los ecosistemas y de la biodiversidad impide la construcción de una relación de sociedad y naturaleza basada en el entendimiento de que el bienestar de los seres humanos depende del manejo respetuoso de los atributos y funciones de la biodiversidad.

Quizás sea necesario, entonces, retomar lecturas de la naturaleza diferentes de las que han circulado históricamente, para así construir nuevos imaginarios de naturaleza que hagan posible su conservación y el bienestar de la sociedad. La concepción de la naturaleza como fenómeno colectivo del mundo, como principio que informa acerca de su funcionamiento o como inspiración, guía y fuente de autoridad, son oportunidades que es preciso explorar en la coyuntura de la crisis ambiental global. 
El diálogo entre las sociedades y los paisajes hace posibles múltiples formas de entender la naturaleza y ofrece la oportunidad de establecer nuevas relaciones con ella a partir de las dinámicas que tienen lugar entre las culturas territoriales y sus paisajes. La construcción de nuevos imaginarios de naturaleza debería considerar al menos tres grandes elementos: la admisión de que la supervivencia y el bienestar humanos dependen de la funcionalidad de los ecosistemas; la inclusión de lecturas de la naturaleza adicionales a la de la ciencia occidental, y la comprensión del papel de las formas de apropiación de la naturaleza en la formación y consolidación de identidades territoriales.

\section{Conflicto de intereses}

Ninguno que declarar.

\section{Referencias}

Álvarez-Munarriz, L. (2011). The Cultural Landscape Concept. AIBR, Revista de Antropología Iberoamericana. 6: 63-86.

Andrade, G. I. \& Castro, L. G. (2012). Degradación, pérdida y transformación de la biodiversidad continental en Colombia, invitación a una interpretación socioecológica. Ambiente y Desarrollo. 16 (30): 53-71.

Andrade, G. I., Chaves, M. E., Corzo, G., Tapia, C. (Editores) (2018). Transiciones socioecológicas hacia la sostenibilidad: gestión de la biodiversidad en los procesos de cambio de uso de la tierra en el territorio colombiano. Bogotá: Instituto de Investigación de Recursos Biológicos Alexander von Humboldt. 220 p.

IPBES. (2018). Las contribuciones de la diversidad biológica y la naturaleza continúan en peligroso declive, alertan los científicos | IPBES. Fecha de consulta: 3 de enero de 2019. Disponible en: https://www.ipbes.net/news/comunicadode-prensa-las-contribuciones-de-la-diversidadbiol\%C3\%B3gica-y-la-naturaleza-contin $\% \mathrm{C} 3 \%$ BAan-.

Butchart, S. H. M., Visconti, P., Hilton-Taylor, C. (2018). Riesgo de extinción: la lista roja de especies amenazadas de la UICN. In Informe Planeta Vivo 2018: apuntando más alto. WWF, p. 104-105.

Carrizosa, J. (2014). Colombia Compleja. Jardín Botánico de Bogotá José Celestino Mutis. Instituto de Investigación de Recursos Biológicos Alexander von Humboldt, Bogotá, D.C.

Ceballos, G., Ehrlich, P. R., Barnosky, A. D., Garcia, A., Pringle, R. M., Palmer, T. M. (2015). Accelerated modern humaninduced species losses: Entering the sixth mass extinction. Science Advances. 1: e1400253-e1400253.

Chapin, F. S., Power, M. E., Pickett, S. T. A., Freitag, A., Reynolds, J. A., Jackson, R. B., Lodge, D. M., Duke, C., Collins, S. L., Power, A. G., Bartuska, A. (2011). Earth stewardship: Science for action to sustain the human-earth system. Ecosphere. 2: 1-20.

Comisión Mundial sobre el Medio Aambiente y el Desarrollo CMMAD. (1987). Informe de la Comisión Mundial sobre el Medio Ambiente y el Desarrollo. Fecha de consulta: 15 de diciembre de 2018. Disponible en: http://www.ecominga. uqam.ca/PDF/BIBLIOGRAPHIE/GUIDE_LECTURE_1/ CMMAD-Informe-Comision-Brundtland-sobre-MedioAmbiente-Desarrollo.pdf.

Coates, P. (1988). Nature, western attitudes since ancient times. University of California Press, Berkeley - Los Angeles. 246 p.
Deinet, S., McRae, L., Freeman, R. (2018). Indicador de población: el índice planeta vivo. En: Grooten, M. \& Almond, R. (Editores): Informe Planeta Vivo 2018: apuntando más alto. WWF, Gland, Switzerland. p. 90-95.

Earth Charter Initiative - ECI (2000). La Carta de la Tierra. Fecha de consulta: 27 de diciembre de 2018. Disponible en: http://cartadelatierra.org/invent/images/uploads/Text\%20 in\%20Spanish.pdf

Etter, A., Andrade, Á., Amaya, P., Arévalo, P. A. (2016). La lista roja de los ecosistemas terrestres de Colombia. En Biodiversidad 2015. Estado y tendencias de la biodiversidad continental de Colombia. Primera edición. Instituto de Investigación de Recursos Biológicos Alexander von Humboldt. p. 29-30.

Grooten, M. \& Almond, R. (Editores) (2018). Informe Planeta Vivo 2018: apuntando más alto. WWF, Gland, Switzerland. $144 \mathrm{p}$.

Guhl-Nannetti, E. \& Leyva, P. (2015). La gestión ambiental en Colombia, 1994-2014: ¿un esfuerzo insostenible? FESCOL-Foro Nacional Ambiental-QUINAXI.

Hoegh-Guldberg, O., Jacob, D., Taylor, M., Bindi, M., Brown, S., Camilloni, I., Diedhiou, A., Djalante, R., Ebi, K. L., Engelbrecht, F., Guiot, J., et al. (2018). Impacts of $1.5^{\circ} \mathrm{C}$ of Global Warming on Natural and Human Systems. pp. 175-311 In: In: Masson-Delmotte, V., P. Zhai, H.-O. Pörtner, D. Roberts, J. Skea, P.R. Shukla, A. Pirani, W. Moufouma-Okia, C. Péan, R. Pidcock, S. Connors, J.B.R. Matthews, Y. Chen, X. Zhou, M.I.Gomis, E. Lonnoy, T.Maycock, M.Tignor, and T. Waterfield (eds.): Global Warming of $1.5^{\circ} \mathrm{C}$. An IPCC Special Report on the impacts of global warming of $1.5^{\circ} \mathrm{C}$ above pre-industrial levels and related global greenhouse gas emission pathways, in the context of strengthening the global response to the threat of climate change, sustainable development, and efforts to eradicate poverty.

Holling, C. S. (2001). Understanding the complexity of economic, ecological, and social systems. Ecosystems. 4: 390-405.

Intergovernmental Panel on Climate Change. (2018). Global warming of $1.5^{\circ} \mathrm{C}$. Special Report: An IPCC special report on the impacts of global warming of $1.5^{\circ} \mathrm{C}$ above pre-industrial levels and related global greenhouse gas emission pathways, in the context of strengthening the global response to the threat of climate change, sustainable development, and efforts to eradicate poverty. Fecha de consulta: 27 de diciembre de 2018. Disponible en: https:// www.ipcc.ch/sr15/ https://www.ipcc.ch/sr15/

Kareiva, P., Watts, S., McDonald, R., Boucher T. (2007). Domesticated nature: Shaping landscapes and ecosystems for human welfare. Science. 316: 1866-1869.

Kennedy, C. M., Oakleaf, J. R., Theobald, D. M., BaruchMordo, S., Kiesecker J. (2019). Managing the middle: A shift in conservation priorities based on the global human modification gradient. Global Change Biology. 25: 1-16. Doi: $10.1111 / \mathrm{gcb} .14549$

Ministerio de Ambiente y Desarrollo Sostenible. (2012). Política nacional para la gestión integral de la biodiversidad y los servicios ecosistémicos (PNGIBSE). Ministerio de Ambiente y Desarrollo Sostenible.

Millennium Ecosystem Assessment Program. (Editor) (2005). Ecosystems and human well-being: Wetlands and water synthesis: A report of the Millennium Ecosystem Assessment. World Resources Institute, Washington, DC. 68 p. 
Mojica, J., Usma, J. S., Álvarez-León, R., Lasso C. A. (Editors) (2012). Libro rojo de peces dulceacuícolas de Colombia 2012. Instituto de Investigación de Recursos Biológicos Alexander von Humboldt, Instituto de Ciencias Naturales de la Universidad Nacional de Colombia, WWF-Colombia y Universidad de Manizales, Bogotá, D.C. 319 p.

Morales-Betancourt, M., Lasso, C. A., Páez, V., Bock, B. (2015). Libro rojo de reptiles de Colombia. Instituto de Investigación de Recursos Biológicos Alexander von Humboldt (IAvH), Universidad de Antioquia, Bogotá, D.C. $258 \mathrm{p}$.

Naranjo, L. G. (2016). La proximidad de los opuestos naturaleza y cultura en el Antropoceno. En: Ruiz, C. E. (Editor). Ciencia y humanismo, 50 años - Revista Aleph. Universidad de Caldas - Universidad Autónoma de Manizales, Manizales. p. 133-152.

Nassauer, J. I. (1992). The appearance of ecological systems as a matter of policy. Landscape Ecology. 6: 239-250.

Nassauer, J. I. (1995). Culture and changing landscape structure. Landscape Ecology. 10: 229-237.

Nogué, J. (2008). Paisaje y sentido de lugar. pp. 207-228. En: DíasFierros, F. \& López-Silvestre, F. (Editores). Olladas críticas sobre a paisaxe. Consello da Cultura Galega, Santiago de Compostela. $312 \mathrm{p}$.

Palacio, G. (2006). Fiebre de tierra caliente, una historia ambiental de Colombia (1850-1930). ILSA, Bogotá. 183 p.

Randazzo-Eisemann, F. (2012). Los imaginarios sociales como herramienta. Imagonautas. 2: 77-92.

Reid, W., Mooney, H. A., Cropper, A., Capistrano, D., Carpenter, S. R., Chopra, K., Dasgupta, P., Dietz, T., Duraiappah, A., Hassan, R., Kasperson, R., et al. (2005). Ecosystems and human well-being - Synthesis: A report of the Millennium Ecosystem Assessment. Island Press, Washington, D.C. 563 p.

Renjifo, L. M., Amaya-Villarreal, A. M., Velásquez-Tibatá, J., Burbano-Girón, J. (2016). Libro rojo de aves de Colombia. Volumen II: ecosistemas abiertos, secos, insulares, acuáticos continentales, marinos, tierras altas del Darién y Sierra Nevada de Santa Marta y bosques húmedos del centro, norte y oriente del país. Editorial Pontificia Universidad Javeriana e Instituto Alexander von Humboldt, Bogotá, D.C. 465 p.

Renjifo, L. M., Gómez, M., Velásquez-Tibatá, J. AmayaVillarreal, A.M., Kattan, G. H., Amaya-Espinel, J.D., Burbano-Girón, J. (Editors) (2014). Libro rojo de aves de Colombia, Volumen I: bosques húmedos de los Andes y la costa Pacífica. Editorial Pontificia Universidad Javeriana e Instituto Alexander von Humboldt, Bogotá, D.C. 202 p.

Rivera, C. C., Duque, A. M., Naranjo, L. G. (2006). De María a un mar de caña: imaginarios de naturaleza en la transformación del paisaje vallecaucano entre 1950 y 1970. Universidad Autónoma de Occidente. 285 p.

Rockström, J., Steffen, W., Noone, K., Persson, A.A., Chapin, F. S., E. F. Lambin, T. M. Lenton, M. Scheffer, C. Folke, H. J. Schellnhuber, et al. (2009). A safe operating space for humanity. Nature. 461: 472-475.
Rodríguez-Becerra, M. \& Espinoza, G. (2002). Gestión ambiental en América Latina y el Caribe. Banco Interamericano de Desarrollo, Washington, D.C. 64 p.

Rozzi, R. (2013). Biocultural ethics: From biocultural homogenization toward biocultural conservation. En: Rozzi, R., Pickett, S. T. A. Palmer, C., Armesto, J. J. \& Callicott, J. B. (Editores). Linking ecology and ethics for a changing world. Springer Netherlands, Dordrecht. p. 9-32.

Sandino, J. C., Aldana-Domínguez, J., Andrade Pérez, G. I., Instituto de Investigación de Recursos Biológicos Alexander von Humboldt. (2011). Biodiversidad y territorio, innovación para la gestión adaptativa frente al cambio global: insumos técnicos para el Plan de Acción Nacional para la Gestión Integral de la Biodiversidad y los Servicios Ecosistémicos. Ministerio de Ambiente, Vivienda y Desarrollo Territorial: Instituto de Investigación de Recursos Biológicos Alexander Von Humboldt, Bogotá. $365 \mathrm{p}$.

Scholte, S. S. K., van Teeffelen, A. J. A., Verburg, P. H. (2015). Integrating socio-cultural perspectives into ecosystem service valuation: A review of concepts and methods. Ecological Economics. 114: 67-78.

Serje, M. (2011). El revés de la nación: territorios salvajes, fronteras y tierras de nadie. Universidad de los Andes. ¿p.?

Soulé, M. E. (2013). The "New Conservation." Conservation Biology. 27: 895-897.

Steffen, W., Grinevald, J., Crutzen, P., McNeill, J. (2011a). The Anthropocene: Conceptual and historical perspectives. Philosophical Transactions of the Royal Society A: Mathematical, Physical and Engineering Sciences. 369: 842-867.

Steffen, W., Persson, Å., Deutsch, L., Zalasiewicz, J., Williams, M., Richardson, K., Crumley, C., Crutzen, P., Folke, C., Gordon, L., Molina, M., et al. (2011b). The Anthropocene: From global change to planetary stewardship. AMBIO. 40: 739-761.

Steffen, W., Richardson, K., Rockstrom, J., , Cornell, S. E., Fetzer, I., Bennett, E. M., Biggs, R., Carpenter, S. R., de Vries, W., de Wit, C. A., Folke, C., et al. (2015). Planetary boundaries: Guiding human development on a changing planet. Science. 347: 1259855-1259855.

Ulloa, A. (2002). Pensando verde: el surgimiento y desarrollo de la conciencia ambiental global. pp. 205-226. En: Palacio, G. \& Ulloa, A. (Editores). Repensando la naturaleza. Universidad Nacional de Colombia - Sede Leticia, Instituto Amazónico de Investigaciones, Instituto Colombiano de Antropología e Historia, Colciencias., Bogotá. 246 p.

Vitousek, P. M., Mooney, H. A., Lubchenko, J., Melillo J. M. (1997). Human domination of earth's ecosystems. Science. 277: 494-499.

Wilson, E. O. (2016). Half-Earth, our planet's fight for life. Liveright Publishing Corporation, New York. 272 p.

WWF-Colombia (2017). Colombia Viva: un país megadiverso de cara al futuro, Informe 2017. WWF Colombia, Cali, Colombia. 162 p. 